\title{
Synthesis, Characterization and Antimicrobial Activities of Some Thiazole Derivatives
}

\author{
E. M. Sharshira ${ }^{1}$, N. M. M. Hamada ${ }^{2, *}$ \\ ${ }^{1}$ Department of Chemistry, Faculty of Science, Alexandria University, Alexandria, Egypt \\ ${ }^{2}$ Department of Chemistry, Faculty of Education, Alexandria University, Alexandria, Egypt
}

\begin{abstract}
In this work a series of thiazoles 9a-1 were prepared by incorporation of pyrazoline ring at position 2 of 2-hydrazinyl-N-(4-phenylthiazol-2-yl) acetamide 8a-c by treating with chalcones 3a-d. The structures of the newly synthesized compounds were determined on the basis of their elemental analyses and spectroscopic data such as IR and HNMR spectra.The antimicrobial activity of isolated heterocyclic compounds was evaluated against Gram-positive, Gram-negative bacteria and fungi. Most of the compounds showed a moderate degree of potent antimicrobial activity.
\end{abstract}

Keywords Chalcones, Thiazoles, Pyrazolines, Antimicrobial

\section{Introduction}

Thiazoles and pyrazoles have been reported to show pharmacological activities. Some of them are used as medicines[1]. According to literature survey, thiazoles were reported to possess antimicrobial[2-5], analgesic[6], antiinflammatory[7], anticonvulsant[8], cardiotonic[9], anticancer[10,11], antitubercular[12] and anthelmintic[13] activities. Antimicrobial activities of some substituted thiazoles are well established because it posses $(\mathrm{S}-\mathrm{C}=\mathrm{N})$ toxophoric unit. Thiazoles have enhanced lipid solubility with hydrophilicity. Thiazoles are easily metabolized by routine biochemical reactions and are non-carcinogenic in nature [14]. In addition, pyrazoles are reported as antimicrobial[15-17], antiviral[18], antipyretic[19], anti-inflammatory[20], antidepressant[21], tranquillizing[22], anticancer[23,24], antihypertensive[25], antiarrhythmi[26], psychoanaleptic[27], anticonvulsant[28] and antidiabetic activities[29].

\section{Results and Discussion}

In our previous studies, we synthesized several pyrazoline derivatives and tested them for their antibacterial and antifungal activities[15,16].

As part of our continuous efforts in This area, a series of some new pyrazoline derivatives containing substituents at 1,3,5-positions were synthesized according to Schemes 13. The starting chalcones 3a-d were prepared in good yields by conventional Claisen-Schmidt condensation by reacting

* Corresponding author:

nagwahamada2002@yahoo.com (N. M. M. Hamada)

Published online at http://journal.sapub.org/ajoc

Copyright (C) 2012 Scientific \& Academic Publishing. All Rights Reserved appropriately substituted benzaldehydes and cyclopropylmethyl ketone[16,30]. 2-aminothiazoles 6a-c[31,32], were obtained in good yields by cyclocondensation of appropriately substituted acetophenones with thiourea in presence of bromine as reported in the literature[33]. Chloroacetamides 7a-c was obtained by reacting of 2-aminothiazoles 6a-c with chloroacetyl chloride in presence of pyridine. The IR of 7a-c showed the presence of bands characteristic for $\mathrm{C}=\mathrm{N}$ at $1631-1642 \mathrm{~cm}^{-1}$ and an amide function at 1661-1671 $(\mathrm{C}=\mathrm{O})$ and $3160-3184 \mathrm{~cm}^{-1}(\mathrm{NH})$. The ${ }^{1} \mathrm{H}$ NMR of 7a-c revealed a broad singlet at $\delta=10.32-10.41 \mathrm{ppm}$ characteristic for a secondary amine group, a multiplet at $\delta=7.11-7.57 \mathrm{ppm}$ for aromatic protons and a thiazolyl- $\mathrm{C}_{5}-\mathrm{H}$ proton as a singlet at 6.49-6.59 ppm. When chloroacetamides 7a-c were heated with hydrazine hydrate in ethanol, hydrazines 8a-c were obtained in a moderate to good yields. The structures of the isolated compounds were determined by spectral methods. The IR of 8a-c revealed characteristic bands for $\mathrm{C}=\mathrm{N}$ at $1637-1641, \mathrm{C}=\mathrm{O}$ at $1664-1669$, primary and secondary amines at $3224-3278$ and $3166-3178 \mathrm{~cm}^{-1}$. The ${ }^{1} \mathrm{H}$ NMR spectra showed the presence of three broad exchangeable singlets at $\delta=9.24-9.29 \mathrm{ppm}, \delta=10.31-10.33 \mathrm{ppm}$ and $\delta=$ 11.45-11.77 ppm characteristic for $-\mathrm{NH}^{-\mathrm{NH}_{2}}$ and $\mathrm{NH}$ protons, while the thiazolyl $-\mathrm{C}_{5}-\mathrm{H}$ and $\mathrm{O}=\mathrm{C}-\mathrm{CH}_{2}$ protons appears at $\delta=6.47-6.55 \mathrm{ppm}$ and $\delta=4.36-4.39 \mathrm{ppm}$, respectively.

The aromatic protons of 8a-c appears as a multiplet at $\delta=$ 7.15-7.66 ppm. Finally, when chalcones $3 \mathrm{a}-\mathrm{d}$ were heated with hydrazines 8a-c in dioxane containing few drops of acetic acid, pyrazoline derivatives 9a-l were obtained. The structures of 9a-l were determined by IR and NMR spectra its IR spectra showed the characteristic bands for $\mathrm{C}=\mathrm{N}$ at 1631-1645 and amide function at 1659-1671 $(\mathrm{C}=\mathrm{O})$ and $3166-3182 \mathrm{~cm}^{-1}(\mathrm{NH})$. The ${ }^{1} \mathrm{H}$ NMR of the pyrazole derivatives 9a-l showed the presence of one broad exchangeable singlet at $\delta=10.31-10.47 \mathrm{ppm}(\mathrm{NH})$, a multiplet at $\delta=$ 
7.12-7.79 ppm (Ar-H's), a singlet at $\delta=6.47-6.60$ (thiazolyl- $\left.\mathrm{C}_{5}-\mathrm{H}\right)$, and a singlet at $\delta=4.30-4.43 \mathrm{ppm}\left(\mathrm{O}=\mathrm{C}-\mathrm{CH}_{2}\right)$. The pyrazoline ring $\mathrm{CH}_{2}$ protons appeared as a pair of doublets of doublets at $\delta=3.07-3.16 \mathrm{ppm}$ and $\delta=3.65-3.81 \mathrm{ppm}$. The $\mathrm{CH}$ proton $\left(\mathrm{H}_{\mathrm{X}}\right)$ appeared as a doublet of doublets at $\delta=$ 5.29-5.42 ppm due to vicinal coupling with the two magnetically non-equivalent protons of the methylene group at position 4 of the pyrazoline ring $\left(J_{\mathrm{AB}}=15 \mathrm{~Hz}, J_{\mathrm{AX}}=3.3 \mathrm{~Hz}\right.$, $J_{\mathrm{BX}}=11 \mathrm{~Hz}$ ). The structure of all newly isolated compounds was fully confirmed by spectral and elemental analyses methods Table $\mathbf{1}$ and $\mathbf{2}$.

\subsection{Antimicrobial Activity}

The in vitro antimicrobial activities of the synthesized compounds were investigated against four pathogenic representative microorganism Staphylococcus aureus ATCC6538P, Pseudomonas aeruginosa ATCC9027, Escherichia coli ATCC8739 and Candida albicans ATCC2091 using Ampicillin, Imipenam and Clotrimazole as standard drugs. Agar well-diffusion method[34] was used for studying the potential activities of these compounds. As shown in Table 3, the antimicrobial effect of the tested compounds was evaluated by measuring the zone diameters and their results were compared with those of well known drugs (standards). Among the tested compounds, it was noticed that compounds 9a-1 demonstrated inhibitory activity more than 8a-c. On the other hand, compounds 8a-c showed more significant antimicrobial activity than those of the chlorothiazole acetamide derivatives 7a-c. Also compounds 7a-c showed more significant antimicrobial activities than those of the aminothiazole derivatives 6a-c. In general, the target compounds 9a-1 showed more significant antimicrobial activity than some known drugs (standards).

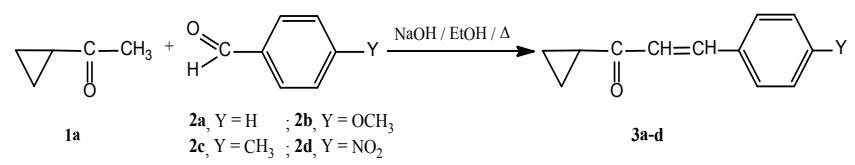

Scheme 1. Synthesis of chalcones 3a-d

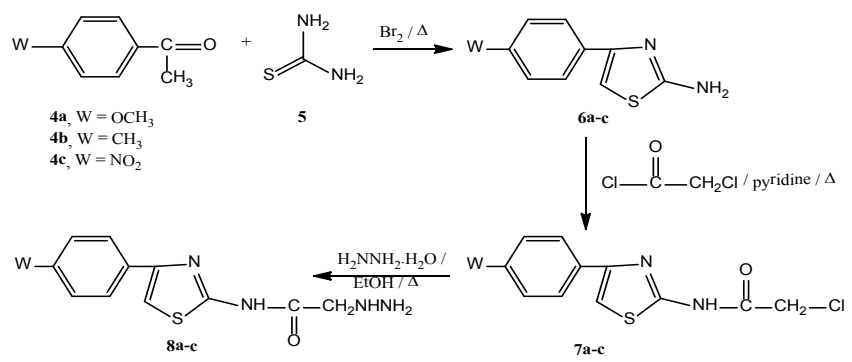

Scheme 2. Synthesis of 2-hydrazinyl-N-(4-phenylthiazol-2-yl)acetamide $8 \mathrm{a}-\mathrm{c}$
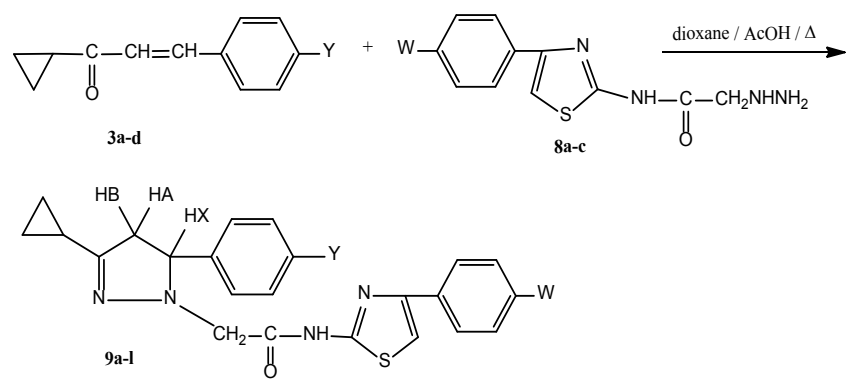

Scheme 3. Synthesis of pyrazoline $9 \mathrm{a}-1$

Table 1. Physical and Analytical Data of Compounds 7a-c, 8a-c and 9a-I

\begin{tabular}{|c|c|c|c|c|c|c|c|c|c|c|c|}
\hline \multirow{2}{*}{ Compound } & \multirow{2}{*}{$\mathrm{Y}$} & \multirow{2}{*}{ W } & \multirow{2}{*}{$\begin{array}{c}\text { M.P. } \\
{ }^{\circ} \mathrm{C}\end{array}$} & \multirow{2}{*}{$\begin{array}{c}\text { Yield } \\
(\%)\end{array}$} & \multirow{2}{*}{$\begin{array}{l}\text { Molecular } \\
\text { Formula }\end{array}$} & \multicolumn{3}{|c|}{ Calculated \% } & \multicolumn{3}{|c|}{ Found \% } \\
\hline & & & & & & $\mathrm{C}$ & $\mathrm{H}$ & $\mathrm{N}$ & $\mathrm{C}$ & $\mathrm{H}$ & $\mathrm{N}$ \\
\hline $7 \mathrm{a}$ & -- & $\mathrm{OCH}_{3}$ & 147 & 81 & $\mathrm{C}_{12} \mathrm{H}_{11} \mathrm{~N}_{2} \mathrm{SClO}_{2}$ & 50.88 & 3.89 & 9.89 & 50.91 & 3.88 & 9.87 \\
\hline $7 b$ & -- & $\mathrm{CH}_{3}$ & 143 & 79 & $\mathrm{C}_{12} \mathrm{H}_{11} \mathrm{~N}_{2} \mathrm{SClO}$ & 53.93 & 4.12 & 10.49 & 53.91 & 4.16 & 10.51 \\
\hline $7 \mathrm{c}$ & -- & $\mathrm{NO}_{2}$ & 152 & 86 & $\mathrm{C}_{11} \mathrm{H}_{8} \mathrm{~N}_{3} \mathrm{SClO}_{3}$ & 44.30 & 2.68 & 14.09 & 44.28 & 2.67 & 14.12 \\
\hline $8 \mathrm{a}$ & -- & $\mathrm{OCH}_{3}$ & 151 & 61 & $\mathrm{C}_{12} \mathrm{H}_{14} \mathrm{~N}_{4} \mathrm{SO}_{2}$ & 51.80 & 5.04 & 20.14 & 51.85 & 5.09 & 20.17 \\
\hline $8 \mathrm{~b}$ & -- & $\mathrm{CH}_{3}$ & 144 & 53 & $\mathrm{C}_{12} \mathrm{H}_{14} \mathrm{~N}_{4} \mathrm{SO}$ & 54.96 & 5.34 & 21.37 & 54.93 & 5.38 & 21.41 \\
\hline $8 \mathrm{c}$ & -- & $\mathrm{NO}_{2}$ & 159 & 63 & $\mathrm{C}_{11} \mathrm{H}_{11} \mathrm{~N}_{5} \mathrm{SO}_{3}$ & 45.05 & 3.75 & 23.89 & 45.07 & 3.72 & 23.92 \\
\hline $9 \mathrm{a}$ & $\mathrm{H}$ & $\mathrm{OCH}_{3}$ & 82 & 73 & $\mathrm{C}_{24} \mathrm{H}_{24} \mathrm{~N}_{4} \mathrm{SO}_{2}$ & 66.67 & 5.56 & 12.96 & 66.71 & 5.58 & 13.01 \\
\hline $9 \mathrm{~b}$ & $\mathrm{H}$ & $\mathrm{CH}_{3}$ & 101 & 69 & $\mathrm{C}_{24} \mathrm{H}_{24} \mathrm{~N}_{4} \mathrm{SO}$ & 69.23 & 5.77 & 13.46 & 69.22 & 5.79 & 13.43 \\
\hline $9 \mathrm{c}$ & $\mathrm{H}$ & $\mathrm{NO}_{2}$ & 104 & 77 & $\mathrm{C}_{23} \mathrm{H}_{21} \mathrm{~N}_{5} \mathrm{SO}_{3}$ & 61.74 & 4.70 & 15.66 & 61.77 & 4.69 & 15.69 \\
\hline $9 d$ & $\mathrm{OCH}_{3}$ & $\mathrm{OCH}_{3}$ & 87 & 49 & $\mathrm{C}_{25} \mathrm{H}_{26} \mathrm{~N}_{4} \mathrm{SO}_{3}$ & 64.94 & 5.63 & 12.12 & 64.91 & 5.62 & 12.11 \\
\hline $9 \mathrm{e}$ & $\mathrm{OCH}_{3}$ & $\mathrm{CH}_{3}$ & 76 & 43 & $\mathrm{C}_{25} \mathrm{H}_{26} \mathrm{~N}_{4} \mathrm{SO}_{2}$ & 67.26 & 5.83 & 12.56 & 67.31 & 5.79 & 12.61 \\
\hline $9 f$ & $\mathrm{OCH}_{3}$ & $\mathrm{NO}_{2}$ & 104 & 64 & $\mathrm{C}_{24} \mathrm{H}_{23} \mathrm{~N}_{5} \mathrm{SO}_{4}$ & 60.38 & 4.82 & 14.68 & 60.37 & 4.84 & 14.71 \\
\hline $9 g$ & $\mathrm{CH}_{3}$ & $\mathrm{OCH}_{3}$ & 98 & 54 & $\mathrm{C}_{25} \mathrm{H}_{26} \mathrm{~N}_{4} \mathrm{SO}_{2}$ & 67.26 & 5.83 & 12.56 & 67.30 & 5.81 & 12.54 \\
\hline $9 \mathrm{~h}$ & $\mathrm{CH}_{3}$ & $\mathrm{CH}_{3}$ & 74 & 57 & $\mathrm{C}_{25} \mathrm{H}_{26} \mathrm{~N}_{4} \mathrm{SO}$ & 69.77 & 6.05 & 13.02 & 69.79 & 6.01 & 13.01 \\
\hline $9 \mathrm{i}$ & $\mathrm{CH}_{3}$ & $\mathrm{NO}_{2}$ & 109 & 59 & $\mathrm{C}_{24} \mathrm{H}_{23} \mathrm{~N}_{5} \mathrm{SO}_{3}$ & 62.47 & 4.99 & 15.18 & 62.46 & 5.02 & 15.21 \\
\hline $9 \mathrm{j}$ & $\mathrm{NO}_{2}$ & $\mathrm{OCH}_{3}$ & 106 & 64 & $\mathrm{C}_{24} \mathrm{H}_{23} \mathrm{~N}_{5} \mathrm{SO}_{4}$ & 60.38 & 4.82 & 14.68 & 60.41 & 4.83 & 14.71 \\
\hline $9 \mathrm{k}$ & $\mathrm{NO}_{2}$ & $\mathrm{CH}_{3}$ & 110 & 60 & $\mathrm{C}_{24} \mathrm{H}_{23} \mathrm{~N}_{5} \mathrm{SO}_{3}$ & 62.47 & 4.99 & 15.18 & 62.44 & 4.96 & 15.20 \\
\hline 91 & $\mathrm{NO}_{2}$ & $\mathrm{NO}_{2}$ & 116 & 69 & $\mathrm{C}_{23} \mathrm{H}_{20} \mathrm{~N}_{6} \mathrm{SO}_{5}$ & 56.10 & 4.07 & 17.07 & 56.13 & 4.10 & 17.04 \\
\hline
\end{tabular}


Table 2. IR and ${ }^{1} \mathrm{H}$ NMR Spectral Data of Compounds 6a-c, 7a-c, 8a-c and 9a-l

\begin{tabular}{|c|c|c|c|c|c|c|c|c|c|c|c|c|c|}
\hline Comp. & & $\mathbb{R} \mathrm{cm}^{-1}$ & $\mathrm{KBr})$ & & & & $\mathrm{NMR}(5 / p p m)^{1}$ & & & Cycloprop & l ring H'S & & \\
\hline & $\begin{array}{l}\mathrm{C}= \\
\mathrm{N}\end{array}$ & $\begin{array}{c}C= \\
0\end{array}$ & $\begin{array}{c}\mathrm{NH} \\
\text { and/or } \\
\mathrm{NH}_{2}\end{array}$ & $\begin{array}{l}\text { Ar-H'S } \\
\text { (II) }\end{array}$ & $\begin{array}{l}\text { Thiazolyl } \\
-C_{-}-\mathrm{H}(\mathrm{s})\end{array}$ & $\begin{array}{l}\text { Pyrazoline-H } \\
\text { dd, } J_{\alpha x}=33 \mathrm{~Hz}, \\
\text { dd, } J_{\alpha}=15 \mathrm{~Hz}\end{array}$ & $\begin{array}{l}\text { Pyrazoline-Hz } \\
\text { dd, } J_{A=}=15 \mathrm{~Hz}, \\
\text { dd, } J_{2 x}=11 \mathrm{~Hz}\end{array}$ & $\begin{array}{l}\text { Pyrazoline-Hx } \\
\text { dd, } \mathrm{J}_{\mathrm{x}}=3.3 \mathrm{~Hz}, \\
\text { dd, } \mathrm{J}_{\mathrm{zx}}=11 \mathrm{~Hz}\end{array}$ & $\begin{array}{c}\mathrm{NH} \text { and/or } \\
\mathrm{NH}_{2}(\mathrm{~s}), \mathrm{D}_{2} \mathrm{O} \\
\text { exchangagble }\end{array}$ & $\begin{array}{l}\mathrm{CH} \\
\text { (II) }\end{array}$ & $\begin{array}{l}2\left(\mathrm{CH}_{2}\right) \\
(\mathrm{I})\end{array}$ & $\begin{array}{c}\mathrm{Ar}-\mathrm{CH}_{2} \\
\mathrm{Ar}-\mathrm{OCH} \text {; } \\
\text { (s) }\end{array}$ & $\begin{array}{c}\mathrm{COCH}_{2} \\
\text { (s) }\end{array}$ \\
\hline $7 a$ & 1633 & 1661 & 3160 & $7.14-7.55$ & 6.49 & - & - & - & 10.32 & - & - & 338 & 422 \\
\hline $7 \mathrm{~b}$ & 1631 & $166 ?$ & 3163 & $7.13-7.57$ & 6.54 & - & - & - & 10.41 & - & - & 2.19 & 431 \\
\hline $7 c$ & 1642 & 1671 & 3184 & $7.11-7.56$ & 6.59 & - & - & - & 1039 & - & - & - & 433 \\
\hline $8 a$ & 1641 & 1669 & 3251,3170 & $7.17-7.60$ & 6.55 & - & - & - & $9,29,10,32,11.45$ & - & - & 3.44 & 439 \\
\hline $8 b$ & 1639 & 1664 & 3224,3178 & $7.15-7.66$ & 6.47 & - & - & - & $926,1031,11.65$ & - & - & 226 & 436 \\
\hline $8 c$ & 1637 & 1668 & 3278,3166 & $7.18-7.59$ & 6.51 & - & - & - & $924,1033,11.77$ & - & - & - & 437 \\
\hline $9 \mathrm{a}$ & 1634 & 1659 & 3177 & $721-7.61$ & 6.47 & 3.07 & 3.74 & 538 & 10.33 & $1.69-2.43$ & $0.69-1.33$ & 334 & 4.43 \\
\hline $9 b$ & 1640 & 1664 & 3166 & $7.14-7.52$ & 6.51 & 3.09 & 3.72 & 537 & 1036 & $1.70-2.43$ & $0.73-1.34$ & 2.17 & 430 \\
\hline $9 c$ & 1643 & 1667 & 3172 & $7.16-7.61$ & 6.57 & 3.08 & 3.81 & 5.40 & 10.41 & $1.73-2.41$ & $0.71-1.36$ & - & 4.41 \\
\hline $9 d$ & 1639 & 1662 & 3173 & $7.27-7.77$ & 6.53 & 3.12 & 3.71 & 5.41 & 10.32 & $1.81-2.43$ & $0.77-1.45$ & 334,329 & 431 \\
\hline $9 e$ & 1637 & 1664 & 3182 & $724 \cdot 7.64$ & 6.47 & 3.13 & 3.75 & 533 & 10.47 & $1.80-2.39$ & $0.71-1.44$ & $322,2.18$ & 433 \\
\hline gf & 1631 & 1669 & 3170 & $7.17-7.72$ & 6.53 & 3.11 & 3.78 & 5.42 & 10.43 & $1.79-2.34$ & $0.72-1.45$ & 333 & 435 \\
\hline $9 g$ & 1635 & 1665 & 3177 & $7.12-7.73$ & 6.56 & 3.07 & 3.69 & 539 & 10.31 & $1.72-2.39$ & $0.75-1.44$ & 326,221 & 438 \\
\hline $9 \mathrm{~h}$ & 1642 & 1661 & 3180 & $7.16-7.79$ & 6.57 & 3.12 & 3.71 & 533 & 10.41 & $1.69-2.39$ & $0.71-1.33$ & 221,226 & 432 \\
\hline $9 \mathrm{i}$ & 1640 & 1669 & 3169 & $7.13-7.72$ & 6.54 & 3.09 & 3.67 & 529 & 1032 & $1.71-2.41$ & $0.72-1.35$ & 2.22 & 430 \\
\hline $9_{j}$ & 1645 & 1671 & 3181 & $7.14-7.79$ & 6.55 & 3.07 & 3.65 & 531 & 10.45 & $1.68-2.43$ & $0.69-1.41$ & 331 & 439 \\
\hline $9 \mathrm{k}$ & 1638 & 1662 & 3176 & $7.18-7.74$ & 6.60 & 3.14 & 3.70 & 539 & 10.32 & $1.67-2.37$ & $0.67-1.33$ & 224 & 437 \\
\hline 91 & 1937 & 1663 & 3178 & $7.17-7.76$ & 6.59 & 3.16 & 3.72 & 536 & 10.41 & $1.70-239$ & $0.70-139$ & - & 4.41 \\
\hline
\end{tabular}

Table 3. The antimicrobial activity of the newly synthesized compounds.

\begin{tabular}{|c|c|c|c|c|}
\hline \multirow{3}{*}{ Tested Compounds and Standards } & \multicolumn{4}{|c|}{ Inhibition zone (mm) } \\
\hline & \multicolumn{4}{|c|}{ Microorganism } \\
\hline & E.Coli & Ps. aeruginosa & S. aureus & C. albicans \\
\hline Ampicillin & - & - & ++ & - \\
\hline Imipenam & ++ & ++ & ++ & - \\
\hline Clotrimazole & - & - & - & ++ \\
\hline $6 a$ & + & - & - & - \\
\hline $6 b$ & + & - & - & - \\
\hline $6 \mathrm{c}$ & + & - & - & - \\
\hline $7 \mathrm{a}$ & ++ & + & + & - \\
\hline $7 b$ & ++ & + & + & - \\
\hline $7 \mathrm{c}$ & ++ & + & + & - \\
\hline $8 \mathrm{a}$ & ++ & + & + & + \\
\hline $8 b$ & ++ & + & + & + \\
\hline $8 c$ & ++ & + & + & + \\
\hline $9 a$ & +++ & ++ & ++ & ++ \\
\hline $9 b$ & +++ & ++ & ++ & ++ \\
\hline $9 \mathrm{c}$ & +++ & ++ & ++ & ++ \\
\hline $9 d$ & +++ & ++ & ++ & ++ \\
\hline $9 \mathrm{e}$ & +++ & ++ & ++ & ++ \\
\hline $9 f$ & +++ & ++ & ++ & ++ \\
\hline $9 \mathrm{~g}$ & +++ & ++ & ++ & ++ \\
\hline $9 \mathrm{~h}$ & +++ & ++ & ++ & ++ \\
\hline $9 \mathrm{i}$ & +++ & ++ & ++ & ++ \\
\hline $9 j$ & +++ & ++ & ++ & ++ \\
\hline $9 \mathrm{k}$ & +++ & ++ & ++ & ++ \\
\hline 91 & +++ & ++ & ++ & ++ \\
\hline
\end{tabular}

+++ Highly sensitive $(21-25 \mathrm{~mm}) ;++$ Fairly sensitive $(16-20) ;+$ Slightly sensitive $(15-10 \mathrm{~mm}) ;-$ Not sensitive.

\section{Experimental}

\subsection{General}

Melting points were taken in open capillary tubes using Electrothermal apparatus 9100 (UK) and are uncorrected. Microanalyses were performed at Faculty of Science, Cairo University, Cairo, Egypt, using an Elementary Vario el III C, $\mathrm{H}, \mathrm{N}, \mathrm{S}$ Analyzer (Germany). IR spectra were recorded using potassium bromide disks on a Spectrum RXI/FT-IR System Perkin Elmer, USA (Faculty of Pharmacy, Alexandria Uni- versity, Alexandria, Egypt). 1H NMR spectra were determined on a Varian EM-390 MHz spectrophotometer, using TMS as internal standard.

\subsection{General Procedure for Preparation of E-l- Cyclo propyl-3-(p-substituted-phenyl)-2-propenones 3a-d}

Compounds $3 \mathrm{a}-\mathrm{d}$ were obtained in a good yield according to published method[16,30]. The physical properties and all the spectral data were as reported in the literature.

\subsection{General Procedure for Preparation of 2-Amino-4- (p- substituted phenyl)-thiazoles 6a-c}


To a mixture consisting of the appropriate acetophenone 4a-d $(10 \mathrm{mmol})$ and thiourea $(10 \mathrm{mmol})$, bromine $(20 \mathrm{mmol})$ was added dropwise during 30 minutes. The reaction mixture was heated on a boiling water bath for 10 hours and water was added to it and again heated until most of the solid has gone into solution. The reaction mixture was filtered on hot and the filtrate was cooled, it was made alkaline with ammonium hydroxide. The product which separated was filtered, washed with water and recrystallized from ethanol to give the desired product as reported previously in literature ${ }^{[33]}$

\subsection{General Procedure for Preparation of 2-Chloro- N-[4- (p-substitutedphenyl)-thiazol-2-yl]-acetamides7a-c}

A solution of 6a-c $(10 \mathrm{mmol})$ in pyridine $(10 \mathrm{~mL})$, chloroacetyl chloride $(12 \mathrm{mmol})$ was added dropwise with continuous stirring. The course of addition was 20 minutes. The formed mixture was heated on a boiling water bath for 3 hours. The mixture was kept to attain room temperature and then poured onto crushed ice. The separated solid was filtered off, washed successively with water, dried and recrystallized from ethanol to give 7a-c. Melting points, elemental analyses, IR and NMR data: see Tables $\mathbf{1}$ and $\mathbf{2}$.

\subsection{General Procedure for Preparation of 2-Hydrazinyl- $\mathrm{N}$-[4-(p-substituted phenyl)-thiazol-2-yl]-acetamides 8a-c}

To a solution of the appropriate 2-chloro-N-[4(p-substituted phenyl) thiazol-2-yl]acetamide 7a-c $(10 \mathrm{mmol})$ in ethanol $(25 \mathrm{~mL})$, hydrazine hydrate $(20 \mathrm{mmol})$ was added. The formed mixture was heated under reflux for 6 hours, and then concentrated under reduced pressure. The formed residue was treated with ice-cold water toseparate a solid product which was filtered, washed successively with water,dried and recrystallized from ethanol to give 8a-c. Melting points, elemental analyses, IR and NMR data: see Tables 1 and 2.

\subsection{GeneralProcedureforPreparation of 2-[3-cyclopropyl-5- (p-substitutedphenyl)-4,5-dihydropyrazol-1-yl]-N-[4- (p-substitutedphenyl)-thiazol-2-yl]-acetamides 9a-l}

A solution of chalcones $3 \mathrm{a}-\mathrm{d}[16,30](10 \mathrm{mmol})$ in dioxane $(10 \mathrm{~mL})$ was refluxed with the appropriate hydrazine $8 \mathrm{a}-\mathrm{c}(10$ $\mathrm{mmol})$ in glacial acetic acid $(1 \mathrm{~mL})$ for 6 hours, then the reaction mixture was poured onto crushed ice and was kept overnight at room temperature. The separated solid was filtered, washed successively with water, dried and recrystallized from methanol. Melting points, IR and NMR data: see Tables 1 and 2.

\subsection{Determination of Antimicrobial Activity}

Cultures of four different microorganisms namely: Escherichia coli (E. coli), Pseudomonas aeruginosa (Ps. aeruginosa), Staphylococcus aureus (S. aureus), Candida albicans (C. albicans) were used to investigate the antimicrobial activities of compounds 6a-c, 7a-c, 8a-c and 9a-l. The antimicrobial activity was assayed biologically using diffusion plate technique. The experiments were carried out by pouring a spore suspension $10^{6}$ colon-forming units (CFU) per $\mathrm{mL}$ of the test strain to $75 \mathrm{~mL}$ of nutrient agar medium at $45{ }^{\circ} \mathrm{C}$ mixed well, and then poured into a $15 \mathrm{~cm}$ sterile metallic Petri plate. The medium was allowed to solidify, and $8 \mathrm{~mm}$ wells were dug with a sterile metallic borer. Then, a DMSO solution of the test sample $(1 \mathrm{~mL})$ at $1 \mathrm{mg} / \mathrm{mL}$ was added to the respective wells. DMSO was served as negative control, and the standard antimicrobial drugs Ampicillin, Imipenam and Clotrimazole were used as positive controls. The layer was allowed to set for $30 \mathrm{~min}$. and incubated at optimum incubation temperature $28 \pm 2{ }^{\circ} \mathrm{C}$. Test organism growth may be affected by the inhibitory action of the test compound and so, a clear zone around the disc appeared as an indication of the inhibition of the test organism growth. The size of clearing zone is proportional to the inhibitory action of the test compound. Measurements were considered after $72 \mathrm{~h}$ for fungi and $24 \mathrm{~h}$ for bacteria. The results are shown in Table 3.

\section{Conclusions}

This work describes simple reactions for the synthesis of new biologically heterocyclic compounds. The antimicrobial activity of these compounds was evaluated against Grampositive, Gram-negative bacteria and fungi. The target compounds 9a-1 showed more significant antimicrobial activity than some known standard drugs on the other hand, most of the compounds showed a moderate degree of potent antimicrobial activity.

\section{REFERENCES}

[1] N. S. Mahajan, S. R. Pattana, R. L. Jadhav, N. V. Pimpodhar, A. M. Manikrao, "Synthesis of some thiazole compounds of biological interest containing mercapta group". Int. J. Chem. Sci. 6(2), 800-806, 2008.

[2] R. V. Ragavan, V. Vijayakumar, N. S. Kumari, "Synthesis and antimicrobial activities of novel 1,5-diarylpyrazoles". Eur. J. Med. Chem. 45(3), 1173-1180, 2010.

[3] El-S. T. Ali, A. M. El. Kazak, "Synthesis and antimicrobial activity of some new 1,3-thiazoles, 1,3,4-thiadiazoles, 1,2,4-triazoles and 1,3-thiazines incorporating acridine and 1,2,3,4-tetrahydroacridine moieties". Eur. J. Chem. 1(1), 6-11, 2010 .

[4] P. Karegoudar, M. S. Karthikeyan, D. J. Prasad, M. Mahalinga, B. S. Holla, N. S. Kumari, "Synthesis of some novel 2,4-disubstituted thiazoles as possible antimicrobial agents". Eur. J. Med. Chem. 43, 261-267, 2008.

[5] P. Vicini, A. Geronkiaki, M. Incerti, F. Zani, J. Dearden, M. Hewitt,"Heteroarylimino-5-benzylidene-4-thiazolidinones analogues of 2-thiazolylimino-5-benzylidene-4-thiazolidinoes with antimicrobial activity: Synthesis and structure-ativity relationship".Bioorg. Med. Chem.16, 3714-3724, 2008.

[6] K. M. Basavaraja, B. Somasekhar, S. appalaraju, "synthesis and biological activity of some 2-[3-substituted- 2-thione- 
1,3,4- thiazole-5-yl]aminobenzothiazoles". Ind. J. Heterocycl. Chem. 18, 69-72, 2008.

[7] T. Karabasanagouda, A.V. Adhikari, D. Ramgopal, G. Parameshwarappa,"Synthesis of some new 2-(4-alkylthiophenoxy)4- substituted-1,3-thiazoles as possible anti-inflammatory and antimicrobial agents" Ind. J. Chem. 47B, 144-152, 2008.

[8] M. A. K. Amine, D. E. Abdel Rahman, Y. A. El-Eryani," Synthesis and preliminary evaluation of some substituted coumarins as anticonvulsant agents". Bioorg. Med. Chem. 16, $5377-5388,2008$.

[9] A. Andreani, M. Rambaldi, A. Leoni, A. Locatelli, R. Bossa, M. Chiericozzi, I. Galatulas, G. Salvatore," Synthesis and cardiotonic activity of imidazo[2,1-b]thiazoles bearing a lactam ring. Eur. J. Med. Chem. 31, 383-387, 1996. B. Jiang, X.- H. Gu, "Syntheses and cytotoxicity evaluation of bis (indolyl) thiazole, bis (indolyl) pyrazinone and bis (indolyl) pyrazine: analogues of cytxic marine bis (indole) alkaloid " Bioorg. Med. Chem. 8, 363-371, 2000.

[10] B. Jiang, X.- H. Gu, "Syntheses and cytotoxicity evaluation of bis (indolyl) thiazole, bis (indolyl) pyrazinone and bis (indolyl) pyrazine: analogues of cytxic marine bis (indole) alkaloid " Bioorg. Med. Chem. 8, 363-371, 2000

[11] T. F. Abbs, F. Reji, S. K. C. Devi, K. K. Thomas, K. G. Sreejalekshmi, S. L. Manju, M. Francis, S. K. Philip, A. Bharathan, K. N. Rajasekharan, "Synthesis and cytotoxicity studies of thiazole analogs of the anticancer marine alkaloid dendrodoine Ind. J. Chem. 47B, 1145-1150, 2008.

[12] Chowki, C. S. Magdum, P. L. Ladda, S. K. Mohite, " Synthesis and antitubercular activity of 6-nitro-2-[4-formyl-3-(substituted phenyl)pyrazol-1-yl]benzothiazoles". Int. J. Chem. Sci. 6(3), 1600-1605, 2008.

[13] K. P. Bhusari, P. B. Khedekar, S. N. Umathe, R. H. Bahekar, R. R.A.Raghu,"Synthesis of 8-bromo-9-substituted-1,3 benzothizolo[5,1-b] 1,3,4-triazoles and their anthelmintic activity "Ind. J Heterocycl. Chem. 9, 275-278, 2000 .

[14] K. Taori, V. J. Paul, H. Luesch, "Structure and activity of largazole, a potent anitproliferative agent from the Floridian marine cyanobacterium Symploca Sp." J. Am. Chem. Soc. 130, 1806-1807, 2008.

[15] E. M. Sharshira, N. M. M. Hamada," Synthesis and in vitro antimicrobial activity of some pyrazolyl-1-carboxamide derivatives. Molecules 16, 7736-7745, 2011.

[16] N. M. M. Hamada, E. M. Sharshira," Synthesis and antimicrobial evaluation of some heterocyclic chalcone derivatives. Molecules 16, 2304-2312, 2011.

[17] R. Kalirajan, S. U. Sivakumar, S. Jubie, B. Gowramma, B. Suresh, "Synthesis and biological evaluation of some heterocyclic derivatives of chalcones." Int. J. Chem. Tech. Res. 1, 27-34, 2009 .

[18] E. Palaska, D. Erol, R. Demirdamar," Synthesis and antidepressant activities of some 1,3,5-triphenyl-2-pyrazolines". Eur. J. Med. Chem. 31, 43-47, 1996.

[19] M. A. Ali, A. A. Siddiqui, M. Shaharyar," Synthesis, structural activity relationship and anti-tubercular activity of novel pyrazoline derivatives". Eur. J. Med. Chem. 42, 268-275, 2007.
[20] M. Amir, H. Kumar, S. A. Khan, Bioorg." Synthesis and pharmacological evaluation of pyrazoline derivatives as new anti-inflammatory and analgesic agents" . Med. Chem.. Lett. 18, 918-922, 2008

[21] A. A. Bilgin, E. Palaska, R. Sunal," Studies on the synthesis and antidepressant activity of some-1-thiocarbamoyl-3,5-diphenyl2-pyrazolines" Arzneim. Forsch. Drug Res. 43, 1041-1044, 1993.

[22] E. Palaska, M. Aytemir, I. T. Uzbay, D. Erol," Synthesis and antidepressant activities of some 3,5-diphenyl-2-pyrazolines. Eur. J. Med. Chem. 36, 539-543, 2001

[23] E. Nassar, "Synthesis, (in vitro) antitumer and antimicrobial activity of some pyrazoline, pyridine and pyrimidine derivatives linked to indoles moiety " J. Am. Sci, 6, 463-471, 2010 .

[24] D. Azarifar, M. Shaebanzadeh, "Synthesis and characterization of new 3,5-dinaphthyl substituted 2-pyrazolines an study of their antimicrobial activity" Molecules 7, 885-895, 2002.

[25] H. Dmytro, Z. Borys, V. Olexandr, Z. Lucjusz, G. Andrzej, L. Roman,"Synthesis of novel thiazolone-based compound containing pyrazoline moiety and evaluation of their anticancer activity". Eur. J. Med. Chem. 44, 1396-1404, 2009.

[26] M. S. Mui, B. N. Siew, A. D. Buss, S. C. Crasta, L. G. Kah, K. L. Sue, "Synthesis of N-1 acidic functionality affording analogues with enhanced antiviral activity against HIV" Bioorg. Med. Chem. Lett. 12, 679-699, 2002

[27] S. S.Parmar, B. R. Pandey, C. Dwivedi, R. D. Harbison, "Anticonvulsant activity and monoamine oxidase inhibitory properties of 1,3,5-trisubstituted pyrazolines" J. Pharm. Sci. 63, 1152-1155, 1974.

[28] A. A. Siddiqui, M. A. Rahman, M. Shaharyar, R. Mishra, "Synthesis and anticonvulsant activity of some 3,5-diphenyl-2-pyrazoline-1-carboxamide derivatives" Chem. Sci. J. 8, 1-10, 2010 .

[29] N. Soni, K. Pande, R. Kalsi, T. K. Gupta, S. S. Parmar, J. B. Barthwal, Inhibition of rat brain monoamine oxidase and succinic dehydrogenase by anticonvulsant pyrazolines "Res. Commun. Pathol. Pharmacol. 56, 129-132, 1987

[30] M. E. Elba,"debromination of erythro-2,3-dibromo-1- cyclopropyl-3-p-substituted phenyl)-1-propanones" J. Pharm. Sci. 9, 43-46, 1995.

[31] Yang-i Lin, C. M. Seifert, S. M. Kang, J. P. Dusza, S. A. Lang Jr, "The synthesis of substituted 2-aminothiazoles" J. Heterocyclic Chemistry. 16 (7), 1377-1383, 1979.

[32] G. Saravanan, V. Alagarsamy, T.G.V. Pavitra, G. C. Kumar, Y. Savithri, L. Naresh, P. Avinash, Synthesis, characterization and anti- microbial activities of novel thiazole derivatives" Int. J. of Pharma and Bio Sciences. 1(3), 1-8, 2010.

[33] S. R. Pattan, R. L. Hullolikar, N. S. Dighe, B. N. Ingalagi, M. B. Hole, V. M. Gaware, P. A. Chavan, "Synthesis and evaluation of some new phenyl thiazole derivatives for their anti- inflammatory activities" J. Pharm. Sci. \& Res. 1(4), 96-102, 2009.

[34] Z. Schelz, J. Molnar, J. Hohmann, "antimicrobial and antiplasmid activities of essensial oils" . Fitoterapia. 77, 279-285, 2006. 